\title{
Clinical pearls to managing men's health conditions during the COVID-19 pandemic
}

\author{
Luke Witherspoon, $M D^{1}$; Ryan Fitzpatrick, $M D^{2}$; Premal Patel, $M D^{3}$; Ryan Flannigan, $M D^{4}$; Matthew T. Roberts, $M D^{\prime}$; \\ Yonah Krakowsky, MD2; Jeffrey D. Campbell, $M D^{5}$; John Grantmyre, MD'; Gerald B. Brock, MD'; \\ S. Larry Goldenberg, $M D^{4}$; Keith A. Jarvi, $M D^{2,7}$
}

'Division of Urology, Department of Surgery, The Ottawa Hospital and University of Ottawa, Ottawa, ON, Canada; ${ }^{2}$ Division of Urology, Department of Surgery, Mount Sinai Hospital, Toronto, ON, Canada; ${ }^{3}$ Section of Urology, University of Manitoba, Winnipeg, MB, Canada; ${ }^{4}$ Department of Urologic Sciences, University of British Columbia, Vancouver, BC, Canada; ${ }^{5}$ Department of Surgery, Division of Urology Western University, London, ON, Canada; 'Department of Urology, Faculty of Medicine, Dalhousie University, Halifax, NS, Canada; ${ }^{7}$ Institute of Medical Sciences, University of Toronto, Toronto, ON, Canada

Cite as: Can Urol Assoc J 2020;14(5):E161-6. http://dx.doi.org/10.5489/cuai.6631

\section{Introduction}

In late 2019, a novel coronavirus (Sars-CoV-2) was identified in Wuhan, China. ${ }^{1}$ Subsequently, the rapid spread of the coronavirus disease 2019 (COVID-19) around the world has resulted in the declaration of a pandemic by the World Health Organization, ${ }^{2}$ with resulting massive changes in societal behaviors.

COVID-19 has had a devastating impact on many of those afflicted with the condition, but the pandemic has also had a dramatic effect on healthcare delivery for those patients without COVID-19. In preparation for expected increased hospital resource needs, most centers have limited or cancelled all elective surgical procedures. Furthermore, in accordance with new social distancing norms, clinical encounters for non-emergent patients have shifted, when possible, to virtual visits.

Urologic surgical care in Canada has undergone a substantial shift to only offering surgery for emergencies and urgent oncology cases. Most of the "bread-and-butter" urology, including the entire subspecialty of men's health and andrology, does not qualify as emergent or even urgent care. Surgeries have been postponed indefinitely for most men requiring intervention for the management of infertility, sexual health, voiding dysfunction, and chronic urological pain.

Thankfully, men's health is an ideal field for virtual care (either by telephone or by video calling) given the many medical and patient education options for treatment. There have been several studies across a variety of specialties that appear to show that virtual visits do not worsen outcomes, and in some cases even improve care. ${ }^{3-7}$ Two studies within the field of urology have been completed showing no adverse outcomes in a general urology virtual followup clinic and in a virtual clinic focused on renal colic.8,9 With the fluid nature of a pandemic scenario, it is unclear when we will once again have access to surgical and clinical resources to provide the standard care for our patients. As a result, we aimed to bring together some of Canada's urologic leaders in men's health to provide pearls and pitfalls regarding the management of common men's health conditions during the pandemic using the virtual care model.

A group of urologists from across Canada with expertise in the area of men's health were invited to provide expert commentary on management of some common men's health conditions during the COVID-19 pandemic. Each of the experts on the panel was provided with a set of standardized written questions (Table 1 ) to answer. The answers were edited by authors LW, RF, and $\mathrm{KJ}$ to fit into a standardized report. The goal of this manuscript is to provide expert guidance to our urology colleagues on how to safely provide virtual care for patients with some of the common men's health conditions, and to provide a resource to our primary care colleagues to understand the options available to the consultant urologist assessing these patients.

\section{Erectile dysfunction}

\section{(Authors: Dr. Jeffrey Campbell, Dr. Gerald Brock)}

Erectile dysfunction (ED) is the recurrent and persistent inability to achieve and/or maintain an erection adequate to permit sexual performance for at least three months. ${ }^{10} \mathrm{ED}$ affects more than $50 \%$ of men between the ages of 40 and 70 years and rates rise over $70 \%$ for patients above $70 .{ }^{11}$

Serious conditions to screen for: Peyronie's disease (PD), cardiovascular disease, neurologic disorders, unstable mental health. 


\section{Table 1. Question's provided to manuscript authors}

1

Is this condition adequately treated with virtual visits, or should this be prioritized for in-person consultations?

2 How has your workup of this patient changed in the era of COVID? What is your current recommended workup for this patient?

3 Are there any useful adjuncts for assessment that can be done at home, that would typically be done in-office?

$4 \quad$ What is your current treatment strategy for this patient? Is there anything a patient can do to optimize their condition at home while awaiting more definitive therapy?

5 What are the dangers/red flags a physician must assess for if providing virtual care for this condition?

6 What documentation do you suggest for the virtual visits?

Virtual visit: For most men complaining of ED, a virtual assessment offers the opportunity to complete a standard consult in a timely fashion. A thorough history is essential to explore the underlying pathophysiology for a patient's ED. The etiology of $\mathrm{ED}$ is often multifactorial and a clinician must explore neurological, vascular, psychological, anatomical, hormonal, and medication-related causes. Additionally, differentiating between hypoactive sexual desire, ejaculatory dysfunction, and PD is best done through careful history. Physical examination has a limited role in the evaluation and management of $E D$ and, therefore, this condition lends itself well to virtual visits. Including the partner (if applicable) in the virtual visit can help elucidate their overall treatment goals more easily.

Suggested investigations: Sexual Health Inventory for Men (SHIM)/International Index of Erectile Function (IIEF) questionnaire, fasting glucose or hemoglobin A1C, lipid profile, testosterone and hormone profile (if warranted). ${ }^{10}$

Treatment: Identifying reversible causes and suggesting lifestyle modifications (smoking cessation, medication changes, exercise, etc.) should be considered first. Phosphodiesterase type 5 inhibitors (PDE5i) remain first-line medical treatment barring contraindications. Patients can perform trials of PDE5is and record their side effects, degree of rigidity, and erection duration. If trials of PDE5i fail in those with a normal hormonal profile, we may suggest the use of a vacuum device or intracavernous injections $(\mathrm{ICI})$, which may require an in-person visit. Surgical interventions for ED are not emergent and will be put off until we can proceed with elective surgery, however, detailed surgical patient information and consent can be carried out virtually. Patients can view YouTube $^{\odot}$ videos or device manufacturer websites, allowing them to gain insight into the various treatment options available. Patients with a psychogenic component to their ED can be referred to local sex counsellors or psychotherapists.

Followup: Poorly treated ED can result in a significant decline in both patient and partner-reported quality of life, however, there is no need for emergent assessment or treatment during the public health crisis. ${ }^{12,13}$ Although rare, patients with priapism due to $\mathrm{ICI}$ treatment need to be assessed and treated despite the COVID-19 pandemic. In-person followups are not urgent and can be arranged in 6-12 months. Patients interested in surgical interventions can be triaged once elective surgery resumes.

\section{Male factor infertility}

\section{(Author: Dr. Keith Jarvi)}

Infertility or subfertility affects $15 \%$ of Canadian couples, with male factor contributing in up to $50 \%$ of cases. ${ }^{14}$ If male factor is present, it is almost always defined by the presence of an abnormal semen analysis, although male factor may play a role even in the presence of a normal semen analysis. ${ }^{15,16}$

Serious conditions to screen for: Testicular masses, sperm banking prior to cancer therapies.

Virtual visit: For most men with infertility, a virtual assessment can be offered as a convenient and safe means to expedite care. An in-person assessment is typically not considered medically urgent except for rare cases, such as testicular masses or need for urgent sperm banking prior to chemotherapy.

A thorough history helps identify possible causes of male factor infertility, as well as understand a couple's sexual habits and treatment goals. The physical examination is not required to initiate investigations and some therapies. Investigations, such as scrotal ultrasonography, can be used to work up any identified anomalies and represents a useful substitute for physical examination if it is available. At least two properly collected semen analyses are required and can be ordered virtually. ${ }^{14}$ Testing, such as hormone profiles and genetic testing, could also be ordered based on the history and other testing.

The initial workup can take months to complete and any medical intervention requires at least three months before benefit can be observed, given the known duration of spermatogenesis. Therefore, initiating assessment and possible treatments virtually can help move these patients along the care pathway effectively until they can be seen physically once the pandemic has resolved. This is especially beneficial for couples where infertility is a time-sensitive manner, such as advanced female partner age. Patients should be counselled that the physical examination is critical to the workup and management of infertility and, therefore, a virtual visit is not a perfect replacement for a clinical encounter but is being used to expedite their care. 
Suggested investigations: Semen analysis. Abnormal semen parameters typically require additional testing, such as a hormone profile (follicle-stimulating-hormone [FSH], luteinizing hormone $[\mathrm{LH}]$, testosterone, and estradiol) and scrotal ultrasound. Further workup, including genetic testing (karyotype and Y-microdeletion assay), transrectal ultrasound, and post-ejaculatory urinalysis can be ordered based on initial investigations as per Canadian Urological Association (CUA)/ American Urological Association (AUA) guidelines. ${ }^{14,16}$

Treatment: Lifestyle recommendations (physical exercise, smoking/marijuana cessation, avoiding hot tubs); sexual counselling regarding timing, frequency, and lubricant use; as well as nutritional supplements can safely be offered to all men. Consultants should also ensure the man's partner is working with a female reproductive specialist and should arrange a consultation if not already done. Medical therapy, such as clomiphene citrate, can safely be offered to appropriately selected patients after virtual discussion of risks and benefits.

Followup: Once the initial workup is completed, clinicians can arrange virtual followup with repeat investigations (semen analysis, hormones) at three month's time to assess for any improvements. Patients, such as those with azoospermia who will require surgical intervention, can be counselled and consented for the procedure virtually so they can be efficiently triaged once elective surgery resumes.

\section{Testosterone deficiency syndrome \\ (Author: Dr. Matthew Roberts)}

Testosterone deficiency syndrome (TDS) can affect men of all ages, have a variety of underlying causes, and present with a myriad of symptoms. While there are long term risks associated with untreated TDS, the short terms risks are likely minimal. However, men must be careful triaged to assess for underlying dangerous medical conditions.

Serious conditions to screen for: Prostate cancer, benign prostatic hyperplasia $(\mathrm{BPH})$, testicular abnormalities, pituitary disorders.

Virtual visit: A careful history should be documented in all men, with a focus on sexual symptoms, mood, concentration and memory, sleep quality, physical performance, and family history of prostatic disease. Portions of the physical examination can be completed by video assessment (general appearance, body habitus, and virilization). Patients can be instructed to perform testicular self-examination and report findings, although the reliability of this method for diagnosing cryptorchidism, testicular atrophy, or scrotal masses is unknown. A scrotal ultrasound could be obtained for any concern if available and can be relied upon to rule out testicular cancers. Clinical judgment must be used to decide on prostate disease risk prior to deferring a digital rectal exam (DRE), as this is mandatory if there is suspicion of prostate disease. ${ }^{17,18}$ Several online tools exist to help estimate prostate cancer risk. ${ }^{19,20}$ Patients with very reassuring prostate-specific antigen (PSA) levels and without risk factors can likely defer their DRE until the pandemic has resolved.

Suggested investigations: Total AM testosterone. If abnormal, obtain confirmatory testosterone measurement along with PSA, FSH, LH, prolactin, and complete blood count (CBC).

Treatment: Intramuscular (IM) testosterone injections have a higher risk of medication-induced erythrocytosis as compared to the other formulations. Furthermore, IM injections usually require at least one in-person visit to the clinic for patient teaching. With limited availability of followup lab tests and requirement for additional patient education visits, we would recommend against IM injections for initial startup therapy. ${ }^{21}$ For patients insistent on this option, there are several good online guides to IM injection technique. ${ }^{22}$ Topical testosterone gel is easily self-administered without the need for a clinic visit, and should be the treatment of choice for new patients, given the current restraints on the healthcare system.

Followup: While most clinicians will perform the first blood work followup at three months after initiating therapy (CBC, PSA, testosterone level), this interval could be extended in patients who have a low baseline PSA and who may be at lower risk of developing erythrocytosis (lower baseline hematocrit [HCT], non-IM therapy). The risk of erythrocytosis in patients treated with topical therapy is highest in the first 3-6 months after initiating treatment and with higher dosing (10 g vs. $5 \mathrm{~g}$ of gel).

Patients on IM therapy are at highest risk of erythrocytosis and, therefore, continued monitoring of HCT is most important in this subgroup. ${ }^{23}$ Consideration should be given to switching these patients, particularly those who are already demonstrating signs of erythrocytosis, to lower doses or to topical agents. ${ }^{24}$

\section{Peyronie's disease \\ (Authors: Dr. Ryan Flannigan, Dr. Yonah Krakowsky)}

Peyronie's disease (PD) involves fibrotic changes to the tunica albuginea of the penile shaft resulting in penile deformity, shortening, erectile dysfunction, and psychological distress. In most cases, a clear diagnosis of PD can be made. However, referrals for penile lumps or nodules may require additional due diligence by the treating urologist to 
ensure more sinister differential diagnoses are not present (i.e., penile or urethral cancer).

Serious conditions to screen for: Penile and urethral cancer.

Virtual visit: A thorough history is critical for understanding the impact of PD, associated conditions, and time course. The active phase of PD is defined as the presence of penile pain with erections and/or penile morphology change observed within the last six months, whereas the chronic phase of PD is defined as the resolution of penile pain and at least six months of no observed penile morphological changes. CUA guidelines suggest that morphological assessment may be performed with digital photos of the erect penis. ${ }^{10}$ Having patients send your clinic digital photos of the erect penis from dorsal and lateral views can be a beneficial tool for assessment. However, it is important for the patient to report the firmness of the erection, as softer erections underestimate deformities. It is also critical to ensure the patient's and the office's receiving emails are secure for transfer of sensitive information. It is best to check with your local guidelines on patient email communication.

Suggested investigations: Validated questionnaires (Peyronie's disease questionnaire [PDQ], IIEF), secure photographs of penile curvature.

Treatment: Among new patients, those in the acute phase of PD may be offered supportive therapies, such as nonsteroidal anti-inflammatory drugs (NSAIDs), or treatment of concomitant erectile dysfunction with PDE5is if no medical contraindications exist. For patients in the chronic stage of $\mathrm{PD}$, treatment planning can prepare the patient to make treatment decisions. While in-office penile injection therapy (i.e., verapamil, interferon, collagenase clostridium histolyticum) and surgical therapy (i.e., penile plication, plaque incision grafting, penile implant) are not currently permitted due to COVID-19, conservative therapies, such as homebased penile traction therapy may be offered to motivated patients. ${ }^{25,26}$ It should be noted that there are no recommended oral therapies. ${ }^{10}$

Followup: Given that PD does not pose an imminent danger to our patients, delayed in-office assessment until resolution of the pandemic is warranted. In the interim, followup should focus on symptom management and treatment planning to expedite care once surgical and clinical services resume. The PDQ serves to track changes and progression in symptom severity, and is particularly useful in this time where assessment is limited by physical distancing. ${ }^{27,28}$

\section{Benign prostatic hyperplasia (Author: Premal Patel)}

Benign prostatic hyperplasia (BPH) and male lower urinary tract symptoms (LUTS) generally have protracted courses, with slow worsening of symptoms over time. Based on prospective studies, the chance of a male with moderate to severe LUTS going into acute urinary retention (AUR) is approximately $0.6-1.8 \%$ per year, with a similar risk of developing infections, bladder stones, or upper tract deterioration. ${ }^{29,30}$ Given the low risk of adverse events and the current pandemic, it is reasonable to monitor symptom progression. However, the urologist treating BPH should focus on ruling out sinister pathologies and identifying men at risk of progressing to adverse events that may require emergent intervention.

Serious conditions to screen for: Prostate cancer, bladder cancer, predictors of AUR, neurogenic bladder.

Virtual visit: The management of BPH can largely be performed virtually. A urinalysis is mandatory, although every effort should be made to obtain a recent $(<6$ month) test to prevent needless visits to laboratories. ${ }^{31}$ A thorough history, with use of validated questionnaires (International Prostate Symptom Score [IPSS]) to identify degree of bother should be done..$^{32}$ Documentation of any gross hematuria, ability to void, current medications, and lack of predominant irritative symptoms must be done to record why ancillary procedures (e.g., cystoscopy) are not needed. PSA is a potential surrogate for prostate size, with elevated PSA and age being two of the strongest predictors of AUR episodes. ${ }^{33}$ Clinicians should recommend their patient begin a voiding diary. This provides invaluable information helping to discern $\mathrm{BPH}$ from other etiologies, such as overactive bladder or nocturia.

Suggested investigations: Urinalysis, PSA, IPSS, voiding diary.

Treatment: Lifestyle modifications are the mainstay of initial management, leading to recommendations to restrict fluids (particularly in the evening); avoid caffeinated beverages, alcohol, spicy foods, and treatment of constipation. It is safe to start most men with a convincing history of BPH on empiric alpha-blocker therapy, although documentation of no history of syncopal/hypotensive episodes should be performed. Similarly, men presenting after failed alpha-blocker therapy can likely be offered a 5-alpha reductase inhibitor. Caution should be applied when considering an antimuscarinic or beta-3 agonist for patients with storage symptoms with an inadequate response to alpha-blocker therapy without prior documentation of a low post-void residual. ${ }^{31}$ With the current operative restrictions, if a man goes into $A \cup R$ and requires catheterization, our preference would be to bring the patient in for clean intermittent catheter $(\mathrm{CIC})$ teaching 
to prevent the need for visits (in office or via community services) every few weeks for catheter changes.

Followup: The field of BPH is fortunate to have well-validated symptom questionnaires, and these should be implemented at each followup visit to attempt to improve any diagnostic accuracy lost in virtual visits. Given the benign nature of the disease, followup can likely be arranged in 6-12 months, hopefully after the resolution of the pandemic.

\section{Scrotal pain \\ (Author: Premal Patel)}

Chronic scrotal pain can often be a very distressing condition to a patient. Given the current pandemic, virtual care for patients with chronic scrotal pain allows assessment of potential etiologies, identifying degree of bother, and reassuring the patient that most causes of chronic scrotal pain are unlikely secondary to a sinister etiology. ${ }^{34}$

Serious conditions to screen for: Testicular cancer, testicular torsion, genitourinary infection.

Virtual visit: While a physical examination is the only way to diagnose the location and type of pain (nociceptive pain arising from the epididymis, testis, musculoskeletal system, or hernia vs. neuropathic pain), a virtual visit may help initiate testing and management for these men. A thorough history should be taken to help the clinician identify a potential etiology. A patient-reported self-exam may also be used, although its validity has never been studied. A symptom diary can be suggested to help document aggravating and alleviating factors. Key documentation of no patient-reported testicular masses, acute onset of pain, testicular swelling, fevers, or purulent drainage must be done. Ultrasound can be used judiciously to followup any concerning findings if available. A urinalysis may be required if there is a history of LUTS or hematuria. If the patient is at high risk for sexually transmitted infections, or has urethral discharge, urethral symptoms, or penile pain, further testing for Nesisseria gonorrhea and Chlamydia trachomatis should be ordered..$^{35}$

Suggested investigations: Urinalysis, screen for sexual transmitted infections, testicular ultrasound for concerning findings on history.

Treatment: All men should be offered conservative management with scrotal support and cold/heat, which can easily be completed at home. Virtual mental health referral may be offered to men, especially if the pain is affecting non-medical aspects of their life. NSAIDs may be considered, if not contraindicated, for those who are NSAID-naive. Alternate agents, such as tricyclic antidepressants or gabapentanoids may also be considered.

Followup: Although often a distressing condition, the etiology of chronic scrotal pain is rarely sinister in origin. As such, virtual care allows the urologist to provide reassurance and recommend conservative and potential medical interventions until a suitable time to perform an in-office assessment.

\section{Discussion}

With the spread of COVID-19 and the need for redistribution of healthcare resources, the field of urology has had to significantly modify its mode of healthcare delivery. While a predominant focus thus far has been on ensuring appropriate triaging of patients with urgent and emergent urological issues, we should not lose sight that many other urological conditions are important for patients and patients don't want to have their care unduly delayed due to the COVID-19 pandemic.

With the pandemic constraints on the urologist's capacity to physically assess the patients, virtual assessment by telephone or video calling represents an alternative means of delivering care in a timely manner. As the expert panellists remarked, virtual care can be used safely to expedite care or, in some cases, to provide complete care for all of the men's health conditions listed. The experts provide a framework to allow our urology colleagues to safely assess men by telephone or video call.

The investigations for all the listed conditions may be initiated following a virtual consultation, and followup visits could also be arranged virtually. Many of the conditions may be treated (at least initially) medically and/or with changes in lifestyle. It should be noted that for all of the conditions described above, there exist CUA patient education handouts (available at: https://www.cua.org/en/patient-information). These represent useful online resources that our patients can be directed to for further information about their conditions and potential therapies.

As the urology community becomes more familiar with virtual appointments out of necessity arising from the pandemic, urologists will begin to understand the benefits and risks of this new model of care. As communication technology improves and patients and doctors become more familiar and comfortable with virtual medical assessments, there will almost inevitably be an expansion of our use of virtual care. The silver lining in this pandemic cloud may be the development and use of expanded virtual patient assessments as a more convenient and safe healthcare delivery strategy. Perhaps in the future, virtual assessments will be routine in the assessment of our patients, providing a reliable and convenient means to provide many initial assessments and followups, replacing the need for many in-person consultations. 


\section{Conclusions}

Many men referred for men's health issues, such as male infertility, sexual health issues, scrotal pain, penile curvature, testosterone deficiency, and voiding dysfunction, may be safely assessed using a virtual visit (telephone or video call) with the subsequent initiation of investigations and therapies.

Competing interests: The authors report no competing personal or financial interests related to this work.

This paper has been peer-reviewed.

\section{References}

1. Zhu N, Zhang D, Wang W, et al. A novel coronavirus from patients with pneumonia in China, 2019. N Engl J Med 2020;382:727-33. https://doi.org/10.1056/NEJMoo2001017

2. WHO Director-General's opening remarks at the media briefing on COVID-19; April 10, 2020. Available at: https://www.who.int/dg/speeches/detail/who-director-general-s-opening-remarks-at-the-mediabriefing-on-covid-19-10-april-2020. Accessed April 12, 2020.

3. Ware P, Ross HJ, Cafazzo JA, et al. Outcomes of a heart failure telemonitoring program implemented as the standard of care in an outpatient heart function clinic: Pretest-post-test pragmatic study. J Med Internet Res 2020;22:e16538. https://doi.org/10.2196/16538

4. Beck CA, Beran DB, Biglan KM, et al. National randomized controlled trial of virtual house calls for Parkinson disease. Neurology 2017;89:1152-61. https://doi.org/10.1212/WNL.0000000000004357

5. Dixon RF, Stahl JE. Virtual visits in a general medicine practice: A pilot study. Telemed. E-Health 2008;14:525-30. https://doi.org/10.1089/tmi.2007.0101

6. Pflugeisen BM, McCarren C, Poore $S$, et al. Virtual visits: Managing prenatal care with modern technotogy. MCN Am J Matern Nurs 2016;41:24-30. https://doi.org/10.1097/NMC.0000000000000199

7. Sultan AA, Acuña AJ, Samuel LT, et al. Utilization of telemedicine virtual visits in pediatric spinal deformity patients: A comparison of feasibility and patient satisfaction at a large academic center. J Pediatr Orthop 2020. [Epub ahead of print]. https://doi.org/10.1097/BP0.0000000000001553

8. Connor MJ, Miah S, Edison MA, et al. Clinical, fiscal, and environmental benefits of a specialist-led virtual ureteric colic clinic: A prospective study. BJU Int 2019;124:1034-9. https://doi.org/10.1111/ bju. 14847

9. Miah S, Dunford C, Edison $M$, et al. A prospective clinical, cost, and environmental analysis of a clinician-led virtual urology clinic. Ann R Coll Surg Engl 2019;101:30-4. hittps://doi.org/10.1308/ resann.2018.0151

10. Bella AJ, Lee JC, Grober ED, et al. 2018 Canadian Urological Association guideline for Peyronie's disease and congenital penile curvature. Can Urol Assoc J 2018;12:E197-209. https://doi.org/10.5489/ cuai.5255

11. Feldman HA, Goldstein I, Hatzichristou DG, et al. Impotence and its medical and psychosocial correlates: Results of the Massachusetts Male Aging Study. J Urol 1994;151:54-61. https://doi.org/10.1016/ S0022-5347(17)34871-1

12. Goldstein I, Fisher WA, Sand M, et al. Women's sexual function improves when partners are administered vardenafil for erectile dysfunction: A prospective, randomized, double-blind, placebo-controlled trial. I Sex Med 2005;2:819-32. https://doi.org/10.1111/i.1743-6109.2005.00147.x

13. Litwin MS, Nied RJ, Dhanani N. Health-related quality of life in men with erectile dysfunction. J Gen Intern Med 1998;13:159-66. https://doi.org/10.1046/i.1525-1497.1998.00050.x

14. Jarvi K, Lo K, Grober E, et al. The workup and management of azoospermic males. Can Urol Assoc J 2015;9:229-35. https://doi.org/10.5489/cuai.3209
15. Barratt CLR, Bï̈rndahl L, De Jonge CJ, et al. The diagnosis of male infertility: An analysis of the evidence to support the development of global WHO guidance-challenges and future research opportunities. Hum Reprod Update 2017;23:660-80. https://doi.org/10.1093/humupd/dmx021

16. Jarow J, Sigman, M, Kolettis, P, et al. The evaluation of the azoospermic male - American Urological Association. Available at: htrps://www.auanet.org/guidelines/azoospermic-male-best-practice-statement. Accessed April 15, 2020.

17. Morales A, Bebb RA, Manjoo P, et al. Diagnosis and management of testosterone deficiency syndrome in men: Clinical practice guideline. CMAJ 2015;187:1369-77. htrps://doi.org/10.1503/cmaj.150033

18. Mulhall JP, Trost LW, Brannigan RE, et al. Evaluation and management of testosterone deficiency: AUA guideline. J Urol 2018;200:423-32. https://doi.org/10.1016/i.juro.2018.03.115

19. PCPT risk calculator. Available at: httrp://www.riskcalc.org/PCPTRC/. Accessed April 12, 2020.

20. Next-generation prostate cancer risk calculator. Available at: https://riskcalc.org/ProstateCA_Screen_ Tool/. Accessed April 12, 2020.

21. Pastuszak AW, Gomez LP, Scovell JM, et al. Comparison of the effects of testosterone gels, injections, and pellets on serum hormones, erythrocytosis, lipids, and prostate-specific antigen. Sex Med 2015;3:165-73. https://doi.org/10.1002/sm2.76

22. Hormone Therapy Self Injection Videos; Planned Parenthood of Greater Texas, Inc. Available at: https://www.plannedparenthood.org/planned-parenthood-greater-texas/self-injection-videos. Accessed April 12, 2020.

23. Ohlander SJ, Varghese B, Pastuszak AW. Erythrocytosis following testosterone therapy. Sex Med Rev 2018;6:77-85. hitps://doi.org/10.1016/i.sxmr.2017.04.001

24. Wang C, Cunningham G, Dobs A, et al. Long-term testosterone gel (AndroGel) treatment maintains beneficial effects on sexual function and mood, lean and fat mass, and bone mineral density in hypogonadal men. J Clin Endocrinol Metab 2004;89:2085-98. https://doi.org/10.1210/ic.2003-032006

25. Chung E, Ralph D, Kagioglu A, et al. Evidence-based management guidelines on Peyronie's disease. J Sex Med 2016;13:905-23. https://doi.org/10.1016/i.jxxm.2016.04.062

26. Nehra A, Alterowitz R, Culkin DJ, et al. Peyronie's disease: AUA guideline. J Urol 2015;194:745-53. https://doi.org/10.1016/i.juro.2015.05.098

27. Coyne KS, Currie BM, Thompson CL, et al. Responsiveness of the Peyronie's disease questionnaire (PDQ). J Sex Med 2015;12:1072-9. https://doi.org/10.1111/ism.12838

28. Hellstrom WJG, Feldman R, Rosen RC, et al. Bother and distress associated with Peyronie's disease: Validation of the Peyronie's disease questionnaire. J Urol 2013;190:627-34. https://doi.org/10.1016/i. juro.2013.01.090

29. MCConnell JD, Roehrborn CG, Bautista OM, et al. The long-term effect of doxazosin, finasteride, and combination therapy on the clinical progression of benign prostatic hyperplasia. N Engl J Med 2003;349:238798. https://doi.org/10.1056/NEJMoa030656

30. Roehrborn CG, Bruskewitz R, Nickel GC, et al. Urinary retention in patients with BPH treated with finasteride or placebo over 4 years. Characterization of patients and uttimate outcomes. The PLESS study group. Eur Urol 2000;37:528-36. https://doi.org/10.1159/000020189

31. Nickel JC, Aaron L, Barkin J, et al. Canadian Urological Association guideline on male lower urinary tract symptoms/benign prostatic hyperplasia (MLUTS/BPH): 2018 update. Can Urol Assoc J 2018;12. https://doi.org/10.5489/cuai.5616

32. Barry MJ, Fowler $\mathrm{FJ}, \mathrm{O}^{\prime}$ leary MP, et al. The American Urological Association symptom index for benign prostatic hyperplasia. J Urol 2017;197:S189-97. https://doi.org/10.1016/i.juro.2016.10.071

33. Roehrborn CG. Acute urinary retention: Risks and management. Rev Urol 2005;7:S31-41.

34. Aljumaily A, Al-Khazraji H, Gordon A, et al. Characteristics and etiologies of chronic scrotal pain: A common but poorly understood condition. Pain Res Manag 2017:e3829168. https://doi.org/10.1155/2017/3829168

35. Jarvi KA, Wu C, Nickel JC, et al. Canadian Urological Association best practice report on chronic scrotal pain. Can Urol Assoc J 2018;12:161-72. htrps://doi.org/10.5489/cuai.5238

Correspondence: Dr. Luke Witherspoon, Division of Urology, The University of Ottawa, Ottawa, ON, Canada; Iwitherspoon@toh.ca; Dr. Keith A. Jarvi, Division of Urology, Mount Sinai Hospital, University of Toronto, Toronto, ON, Canada; keith.jarvi@sinaihealth.ca 